\title{
Bedaquilin: Ein neuer Wirkstoff bei MDR-TBC?
}

Die jährliche Tuberkuloseinzidenz von rund 9 Mio. mit 1,5 Mio. Todesfällen weltweit ist ein immenses globales Problem. Rund 450000 Fälle von multiresistenten Tuberkulosen (MDR-TBC) zeigen selbst hochentwickelten medizinischen Infrastrukturen ihre Grenzen auf. Deshalb liegen große Hoffnungen auf dem ersten völlig neuen TBC-Medikament seit 40 Jahren. Hierzu hat ein internationales Forscherteam um A. H. Diacon et al. nun die Ergebnisse einer Phase-Ilb-Studie vorgelegt. N Engl J Med 2014; 371: 723-732

Die Autoren verabreichten 160 Patienten mit multiresistenter Lungentuberkulose (MDR-TBC) randomisiert und doppelt verblindet über 24 Wochen entweder Bedaquilin oder Placebo. Zusätzlich erhielten alle Patienten eine den WHO-Empfehlungen konforme Kombinationstherapie mit 5 verschiedenen Wirkstoffen. Die Patienten wurden 120 Wochen lang beobachtet. Als primären Endpunkt definierte das Studienprotokoll die Dauer bis zur Sputumkonversion in einer Flüssigkultur.

Die Sputumkonversion trat unter Bedaquilin im Mittel nach 83 Tagen ein und damit deutlich früher als unter Placebo, wo dies nach durchschnittlich 125 Tagen der Fall war (Hazard Ratio 2,44; 95\%-Konfidenzintervall 1,57-3,80, p<0,0001). Die Rate der Sputumkonversion lag nach 24 Wochen mit 79 vs. $58 \%$ ebenso wie nach 120 Wochen mit 62 vs. 44\% unter Verum deutlich höher. Auf der Grundlage der WHO-Definition für die Heilung einer MDR-TBC erwies sich Bedaquilin mit 58 vs. $32 \%$ ebenfalls als deutlich wirksamer als Placebo $(p=0,003)$. Obwohl sich die Gesamtinzidenz unerwünschter Nebenwirkungen nicht unterschied, traten in der Bedaquilin-Gruppe 10 Todesfälle auf, während in der Placebogruppe nur 2 Personen starben.

Die Autoren weisen darauf hin, dass die Mortalität in der Placebogruppe ungewöhnlich niedrig war: In einer Metaanalyse von MDR-Tuberkulosen mit über 9000 Patienten habe diese bei 15\% gelegen.

\section{Fazit}

Die zusätzliche Gabe von Bedaquilin zu einer optimalen 5-fach-Therapie verkürzt bei multiresistenten Lungentuberkulosen die Zeit bis zur Serumkonversion, so die Autoren. Außerdem erhöhe sie die Rate von Serumkonversionen nach 120 Wochen. Wegen der unter Bedaquilin beobachteten Übersterblichkeit raten die Autoren aber dazu, Bedaquilin bis zum Vorliegen aussagekräftiger, größerer Studien nur einzusetzen, wenn es dafür keine wirksame Alternative gibt.

\section{Kommentar zur Studie}

In ihrem Kommentar zur Studie geben E. Cox und K. Laessig von der US-amerikanischen Food and Drug Administration (FDA) zu bedenken, dass 8 der 10 Todesfälle unter Bedaquilin im Mittel 329 Tage nach Beendigung der Therapie eingetreten sind. Trotz der langen Halbwertszeit der Substanz sei deshalb ein kausaler Zusammenhang schwer zu erklären. Wegen seines neuen Wirkmechanismus sei Bedaquilin aber eine Behandlungsoption für Patienten, bei denen alle anderen Therapiestrategien versagt haben.

N Engl J Med 2014; 371: 689-691

\section{Dr. Peter Pommer, Oberammergau}

Epidemiologie

\section{Tuberkulosezahlen nach oben korrigiert}

Die Weltgesundheitsorganisation (WHO) hat ihre Schätzungen zur Anzahl der Tuberkulose-Erkrankungen um fast 500000 nach oben korrigiert. Im Jahr 2013 erkrankten weltweit 9 Mio. Menschen, 2012 waren es noch 8,6 Mio. Infektionen. Die Zahl der Todesopfer sinkt jedoch. Im vergangenen Jahr starben rund 1,5 Mio. Menschen. Laut dem Global Tuberculosis Report 2014 waren 360000 der Verstorbenen HIV-positiv. 2012 forderte die Krankheit 1,3 Mio. Todesopfer. Einer der Hauptfaktoren für die Korrektur der Anzahl der Erkrankungen ist, dass in den einzelnen Ländern Daten genauer erhoben werden. Langfristig gesehen sind die Todesfälle seit 1990 um 45\% gesunken. In diesem Zeitraum ist die Anzahl der Erkrankungen um 1,5\% pro Jahr zurückgegangen. Im Jahr 2013 wurde laut WHO Tuberkulose bei rund 3 Mio. Menschen nicht diagnostiziert. Laut der WHO-Studie erkrankten 2013 die meisten Menschen in Südostasien und im westlichen Pazifik an Tuberkulose. Allein auf Indien entfallen 24\% der Erkrankungen und auf China 11\%. Ein weiteres Viertel der Infektionen trat in Afrika auf, das über die höchste Anzahl von Erkrankungen und Todesfällen in Relation zur Bevölkerung verfügt. Mangelnde finanzielle Mittel erschweren die Bekämpfung. Schätzungen gehen von 8 Mrd. USDollar aus, die jährlich nötig wären. Derzeit fehlen pro Jahr allerdings etwa 2 Mrd. US-Dollar. Im Jahr 2013 entfielen 3,5\% der Neuerkrankungen auf Formen von Tuberkulose, die gegen Medikamente resistent sind. Weltweit dürften rund 5\% der Erkrankungsfälle davon betroffen sind. Laut WHO kommt es jedoch in einigen Regionen der Welt wie in Osteuropa und Zentralasien zu regelrechten Epidemien. In Weißrussland soll bei 35\% der Neuerkrankungen eine Resistenz vorliegen. Derzeit werden aus 100 Ländern Erkrankungen mit hochresistenter Tuberkulose gemeldet. Diese Form ist noch schwerer zu behandeln als die resistente Form. Der Organisation „Ärzte ohne Grenzen“ zufolge ist der Anstieg von resistenter Tuberkulose vor allem in der früheren Sowjetunion Besorgnis erregend.

pressetext Nachrichtenagentur 